Al1104 589682

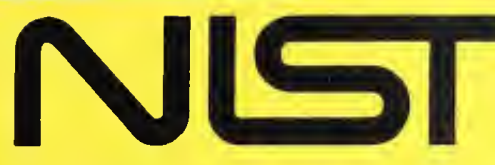

United States Department of Commerce Technology Administration National Institute of Standards and Technology

NISTIR 5033

\title{
Polarimetric Calibration of Reciprocal-Antenna Radars
}

\author{
R.L. Lewis \\ L.A. Muth \\ R.C. Wittmann
}

QC

100

.456

N0. 5033

1995 



\section{Polarimetric Calibration of Reciprocal-Antenna Radars}

R.L. Lewis

L.A. Muth

R.C. Wittmann

Electromagnetic Fields Division

Electronics and Electrical Engineering Laboratory

National Institute of Standards and Technology

Boulder, Colorado 80303-3328

March 1995

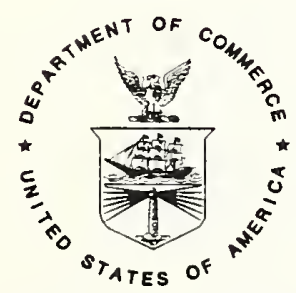

U.S. DEPARTMENT OF COMMERCE, Ronald H. Brown, Secretary TECHNOLOGY ADMINISTRATION, Mary L. Good, Under Secretary for Technology NATIONAL INSTITUTE OF STANDARDS AND TECHNOLOGY, Arati Prabhakar, Director 



\title{
Polarimetric Calibration of Reciprocal-Antenna Radars
}

\author{
R. L. Lewis, L. A. Muth, and R. C. Wittmann
}

\author{
Electomagnetic Fields Division \\ National Institute of Standards and Technology \\ Boulder, CO 80303
}

\begin{abstract}
We discuss how radar target depolarization enhances a radar's crosspolarization contamination, and we present a graphical study of RCS measurement error due to depolarization by an inclined dihedral reflector. Since error correction requires polarimetric RCS measurements, we recommend upgrading singly polarized radars to full polarimetric capability. We present a polarimetric calibration technique that is applicable to reciprocal antenna radars, saves calibration time by requiring a single calibration target, uses simple mathematical expressions, and smoothes measured calibration data to reduce the effects of clutter and noise.
\end{abstract}

Key words: antennas; calibration; dihedral; measurement uncertainty; polarimetric error correction; polarization; radar; RCS; reciprocity.

\section{Introduction}

In this work we graphically demonstrate the errors in radar cross section measurements when target depolarization is ignored, even when the radar has good polarization isolation [1]. 
We then introduce a simplified procedure for calibrating polarimetric radar systems. The radar is modeled as a reciprocal-antenna system coupled to an 'ideal' transmitter/receiver system which has perfect polarization isolation. Assumption of reciprocity reduces the number of unknown parameters and leads to a calibration method which is simpler mathematically and which requires fewer distinct calibration targets than other methods [2] through [6]. Since in a well designed and maintained radar, transmitter/receiver system isolation can be very good compared to antenna isolation, the assumption of an ideal transmitter/receiver is not restrictive; however, relative cross-talk levels should be verified experimentally.

A single standard target, a rotating dihedral, is used. Data is collected as a function of rotation angle and Fourier analysis is applied to reduce the effects of clutter and noise. (A more general approach, recommended for future study, is to characterize a 'nonideal' transmitter/receiver through a separate calibration at the antenna terminals.)

Due to misalignment or physical imperfection, the antenna system will not be perfectly polarized, and many targets have significant potential for depolarizing an incident field and returning a large cross-polarized component. Cross-polarization errors can be ignored only when there is good polarization isolation and when depolarization by the target is minimal; otherwise, the error resulting from failure to correct for polarimetric contamination can be very large.

Depolarized backscatter is readily produced by targets which, for instance, reflect incident radiation more than once before $\cdot$ it is returned; a simple example is an inclined dihedral reflector. Thus, measurement of the RCS of 'stealth' targets, which redirect incident radiation, requires polarimetric correction for accurate results.

\section{Polarization Error in Single-Polarization Radars}

Here, the adjective single-polarization refers to a radar that ideally transmits and receives one polarization at a time; for example, vertical transmit and receive or horizontal transmit and receive. In any practical situation, however, there will always be some polarization contamination. 
The signal $S_{v v}$ that is received by a nonideal vertical single-polarization radar can be expressed in terms of the target's scattering matrix $\mathbf{A}$ as

$$
S_{v v} \propto\left(\begin{array}{ll}
R_{v h} & R_{v v}
\end{array}\right)\left(\begin{array}{cc}
A_{h h} & A_{h v} \\
A_{v h} & A_{v v}
\end{array}\right)\left(\begin{array}{c}
T_{h v} \\
T_{v v}
\end{array}\right) .
$$

The horizontal polarization case follows immediately upon interchanging $h$ and $v$. In eq (1), $R_{v x}$ and $T_{x v}$ denote the radar system's receiving and transmitting characteristics for the $x=h$ (horizontal) or the $x=v$ (vertical) polarization components.

For the case of an ideal vertical polarization system, the cross components $R_{v h}$ and $T_{h v}$ are identically zero, in which case $S$ becomes proportional to $R_{v v} A_{v v} T_{v v}$. Consequently, a nonideal vertical-polarization system has an RCS measurement error (in decibels) of

$$
\mu=20 \log \left|1+\frac{R_{v h} A_{h v} T_{v v}}{R_{v v} A_{v v} T_{v v}}+\frac{R_{v v} A_{v h} T_{h v}}{R_{v v} A_{v v} T_{v v}}+\frac{R_{v h} A_{h h} T_{h v}}{R_{v v} A_{v v} T_{v v}}\right| .
$$

Here, $\mu \equiv 10 \log \left(\sigma_{m} / \sigma_{v}\right)$, where $\sigma_{m}$ is the measured RCS and $\sigma_{v}$ is the ideal RCS. All target and antenna parameters contribute to the error, and each term in eq (2) has a physical interpretation.

In the case of 'weak' depolarization, the term

$$
\frac{R_{v h} A_{h h} T_{h v}}{R_{v v} A_{v v} T_{v v}}
$$

is dominant, which simply means that depolarization by the target is minimal. In this case the error term is just the ratio of the two copolarized fields returned from the target due to the antenna's vertical-polarization channel transmitting and receiving both main and cross components. In the case of 'strong' depolarization, the terms

$$
\frac{R_{v h} A_{h v} T_{v v}}{R_{v v} A_{v v} T_{v v}}+\frac{R_{v v} A_{v h} T_{h v}}{R_{v v} A_{v v} T_{v v}}
$$

are dominant. Here, the error is primarily due to the main and crosscomponent fields incident upon the target giving rise, respectively, to crossand main-component reflections which interact with the radar's cross- and main-component receiving properties to produce an enhanced error term. Thus, the dominant error term is the sum of two depolarized signals from the target divided by the copolarized return. 
If the radar antenna is reciprocal ${ }^{1}$, then $R_{v h} / R_{v v}=T_{h v} / T_{v v}$ [see eq (27)]. If the target is also reciprocal, $A_{h v}=A_{v h}$, eq (1) becomes

$$
S_{v v} \propto\left(R_{v h} A_{h h} R_{v h}+2 R_{v h} A_{h v} R_{v v}+R_{v v} A_{v v} R_{v v}\right) .
$$

From eq (5), we have weak depolarization when

$$
\left|\frac{R_{v h}}{R_{v v}}\right|>>2\left|\frac{A_{h v}}{A_{h h}}\right|
$$

and we have strong depolarization when

$$
\left|\frac{R_{v h}}{R_{v v}}\right|<2\left|\frac{A_{h v}}{A_{h h}}\right| .
$$

As an example, consider the interaction between the radar system and a dihedral reflector. If the line of intersection between the dihedral reflector's two perpendicular planes is oriented at an angle $\theta$ with respect to horizontal, then the dihedral reflector's scattering matrix ${ }^{2}[2,4,7,8,9,10]$ has the form

$$
\mathbf{A}=K_{d}\left(\begin{array}{cc}
\cos 2 \theta & \sin 2 \theta \\
\sin 2 \theta & -\cos 2 \theta
\end{array}\right) \text {. }
$$

Consequently, the received signal $S$ is

$$
S_{v v} \propto R_{v h}^{2} \cos 2 \theta+2 R_{v h} R_{v v} \sin 2 \theta-R_{v v}^{2} \cos 2 \theta,
$$

which gives an RCS error of

$$
\mu=20 \log \left|1-2 \frac{R_{v h}}{R_{v v}} \tan 2 \theta-\left(\frac{R_{v h}}{R_{v v}}\right)^{2}\right| .
$$

Thus, we have weak depolarization when

$$
\left|\frac{R_{v h}}{R_{v v}}\right|>>2 \tan 2 \theta
$$

and strong depolarization when

$$
\left|\frac{R_{v h}}{R_{v v}}\right|<<2 \tan 2 \theta \text {. }
$$

\footnotetext{
${ }^{1}$ We assume that the antenna system has a single transmit/receive port.

${ }^{2}$ Calibration accuracy is limited by the geometrical optics approximation. Estimation of the resulting uncertainty is beyond the scope of this paper.
} 
If $\eta \equiv-20 \log \left|R_{v h} / R_{v v}\right|$, so that $R_{v h} / R_{v v}=q e^{i \alpha}$ with $q \equiv 10^{-\eta / 20}$, then eq (10) becomes

$$
\mu=10 \log \left(\left(1+q^{2}\right)^{2}-4 q^{2} \cos ^{2} \alpha-4 q\left(1-q^{2}\right) \cos \alpha \tan 2 \theta+4 q^{2} \tan ^{2} 2 \theta\right) .
$$

Figures 1 through 6 show the error $\mu$ [from eq (13)] in the measured RCS of a dihedral reflector, as a function of $\theta$ for various values of $R_{v h} / R_{v v}$. These figures demonstrate that assuming ideal polarization can result in large uncertainties, even when the system has relatively good polarization isolation.

Figures 1 and 2 show the error range by presenting the extreme cases of in-phase polarization ratios and out-of-phase polarization ratios $\left(\alpha=0^{\circ}\right.$ or $\alpha=180^{\circ}$ ) for several values of $\eta$. Figures 3 through 6 show the error for several values of $\alpha$ when $\eta$ is fixed. The lowermost scale in each figure gives $-10 \log \left[\sigma_{v}(\theta) / \sigma_{v}(0)\right]$ (in decibels).

The existence of cross-polarization contamination on an RCS range can be demonstrated using a dihedral reflector. Assuming reasonably good alignment of the reflector with respect to the radar, then at an angle of $0^{\circ}$ or $90^{\circ}$ to the horizontal the reflected field will have the same polarization as the incident field, while at $45^{\circ}$ the reflected field will be completely depolarized. The ratio of the received signals from these two cases gives an indication of the system's cross-polarization contamination (see below).

\section{Polarimetric Calibration of Reciprocal-Antenna Radars}

The matrix of received signals measured by a polarimetric radar can be expressed [see eq (1)]

$$
\left(\begin{array}{ll}
S_{h h} & S_{h v} \\
S_{v h} & S_{v v}
\end{array}\right) \propto\left(\begin{array}{ll}
R_{h h} & R_{h v} \\
R_{v h} & R_{v v}
\end{array}\right)\left(\begin{array}{ll}
A_{h h} & A_{h v} \\
A_{v h} & A_{v v}
\end{array}\right)\left(\begin{array}{ll}
T_{h h} & T_{h v} \\
T_{v h} & T_{v v}
\end{array}\right) .
$$

Here, $S_{x y}$ represents a received $x$-polarized signal when a $y$-polarized signal is transmitted, $T_{x y}$ represents an $x$-polarized signal that is transmitted when the radar is set to transmit a $y$-polarized signal, and $R_{x y}$ represents a received $x$-polarized signal due to an incident $y$-polarized signal.

We assume a reciprocal dual-port antenna system. (Two-antenna radars are considered in appendix B). If transmitter and receiver channels are iso- 


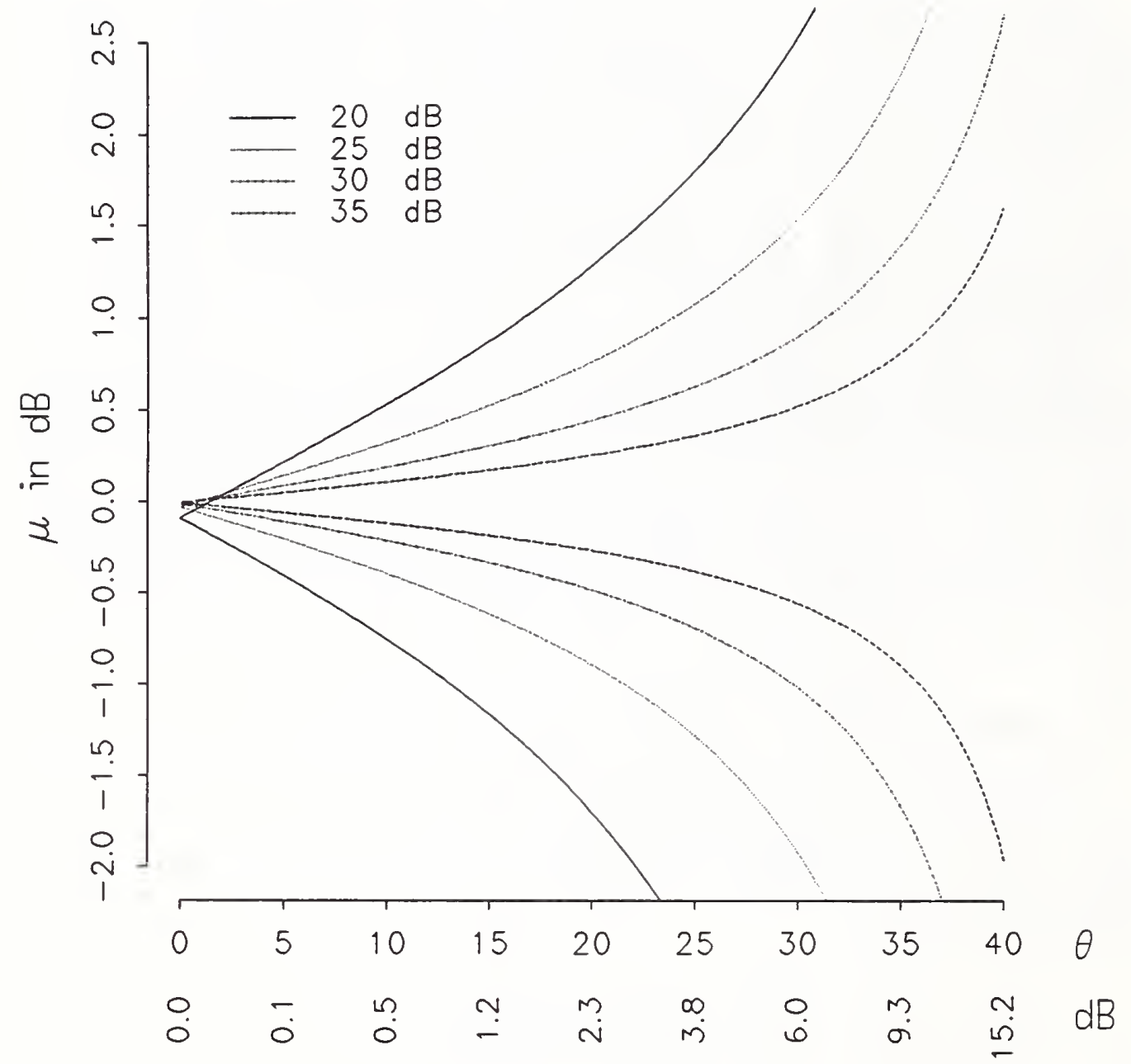

Figure 1: The error $\mu$ in the measured RCS of a dihedral scatterer for $\eta=20$, 25,30 , and $35 \mathrm{~dB}$ (upper curves $\alpha=180^{\circ}$, lower curves $\alpha=0^{\circ}$ ). 


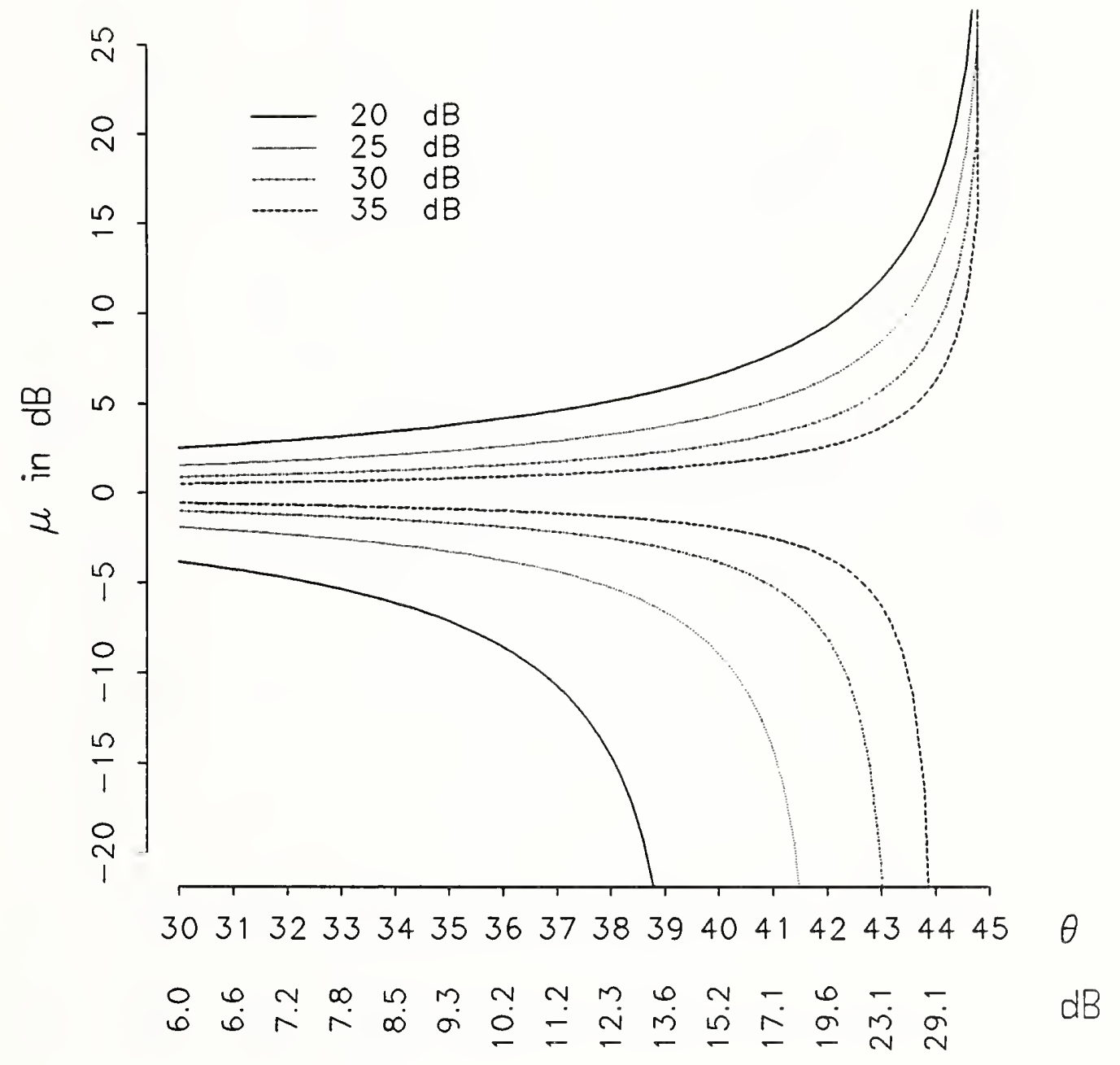

Figure 2: The error $\mu$ in the measured RCS of a dihedral scatterer for $\eta=20$, 25,30 , and $35 \mathrm{~dB}$ (upper curves $\alpha=180^{\circ}$, lower curves $\alpha=0^{\circ}$ ). 


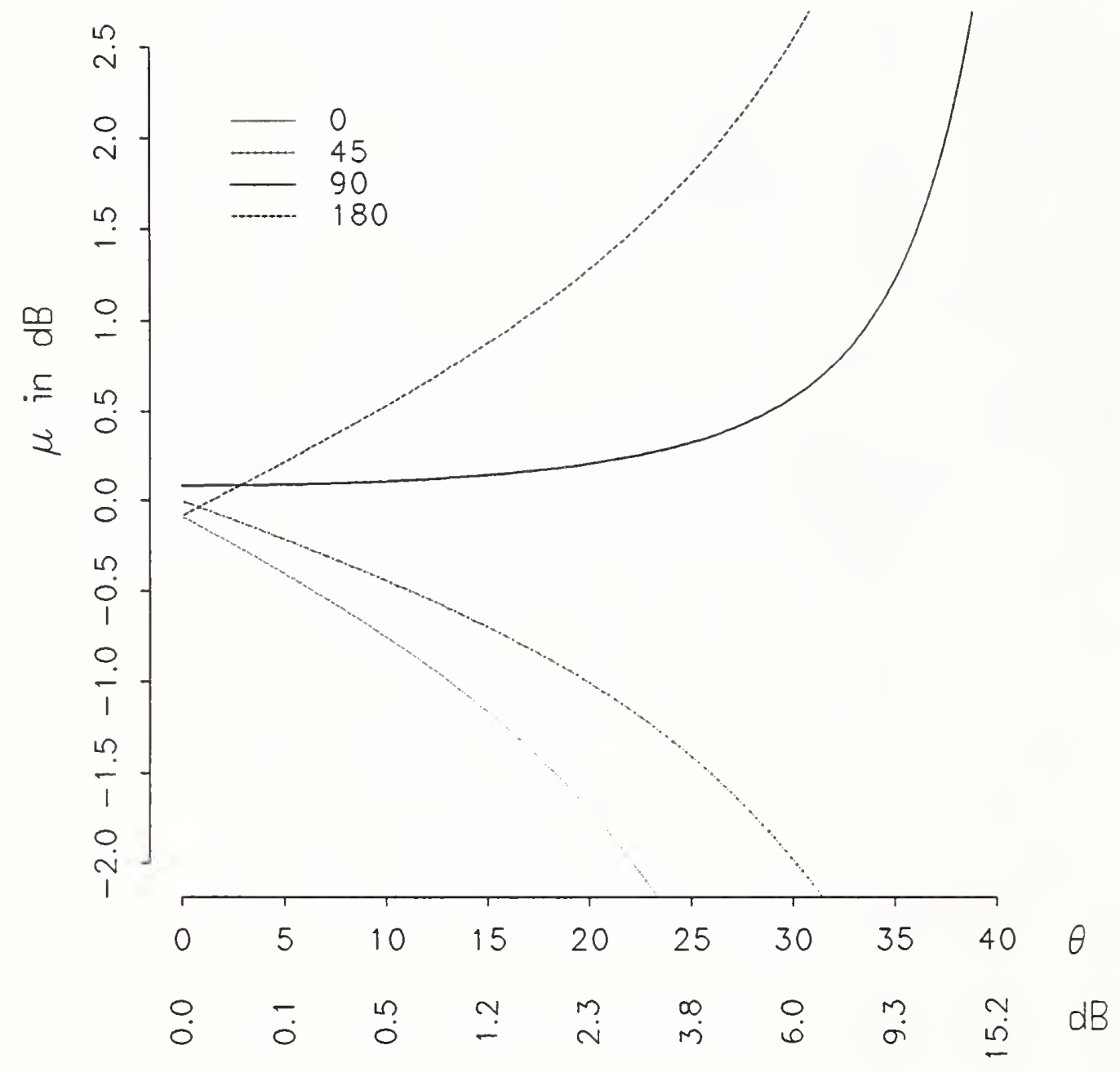

Figure 3: The error $\mu$ in the measured RCS of a dihedral scatterer for $\alpha=0^{\circ}$, $45^{\circ}, 90^{\circ}$, and $180^{\circ}(\eta=20 \mathrm{~dB})$. 


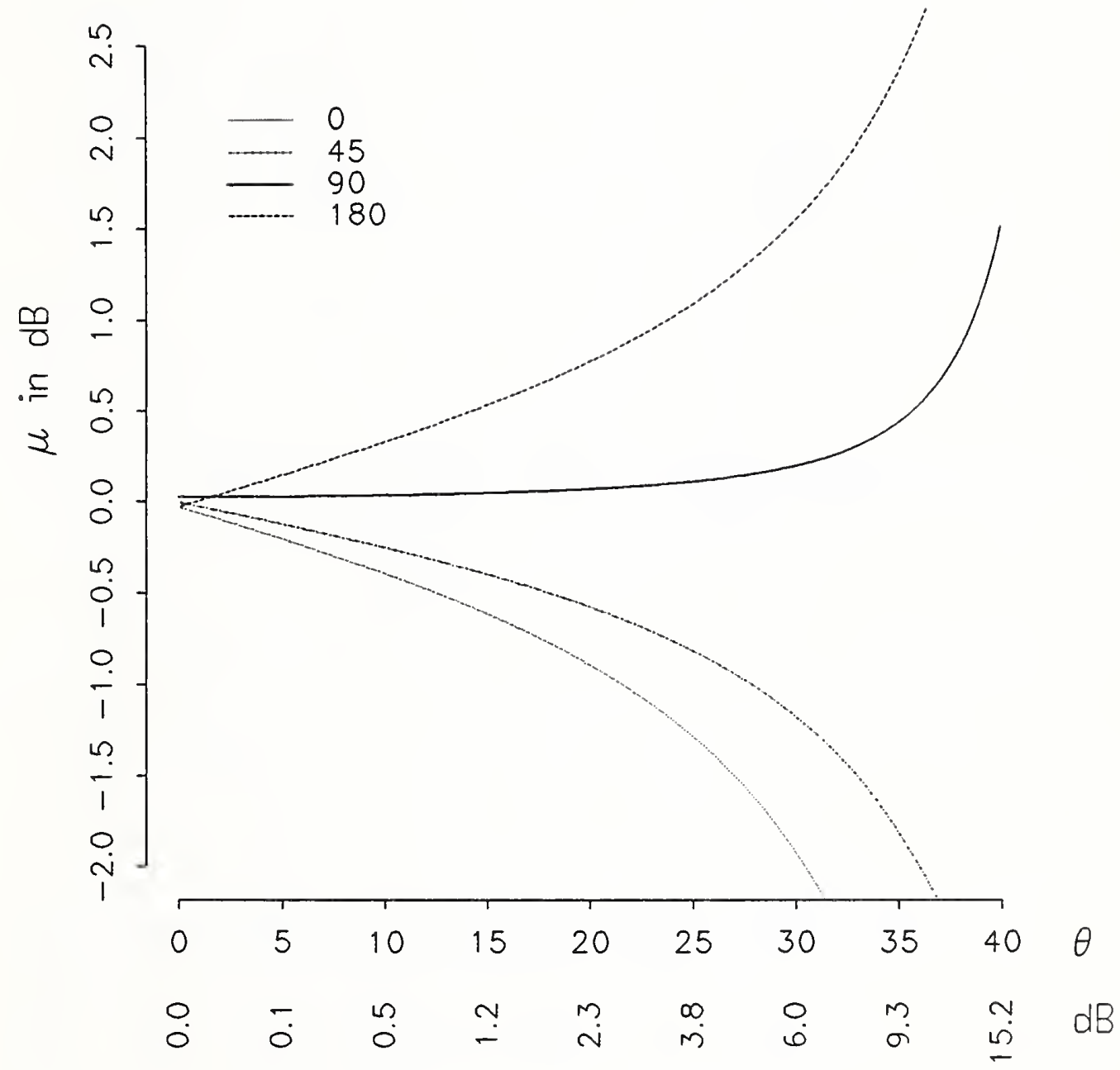

Figure 4: The error $\mu$ in the measured RCS of a dihedral scatterer for $\alpha=0^{\circ}$, $45^{\circ}, 90^{\circ}$, and $180^{\circ}(\eta=25 \mathrm{~dB})$. 


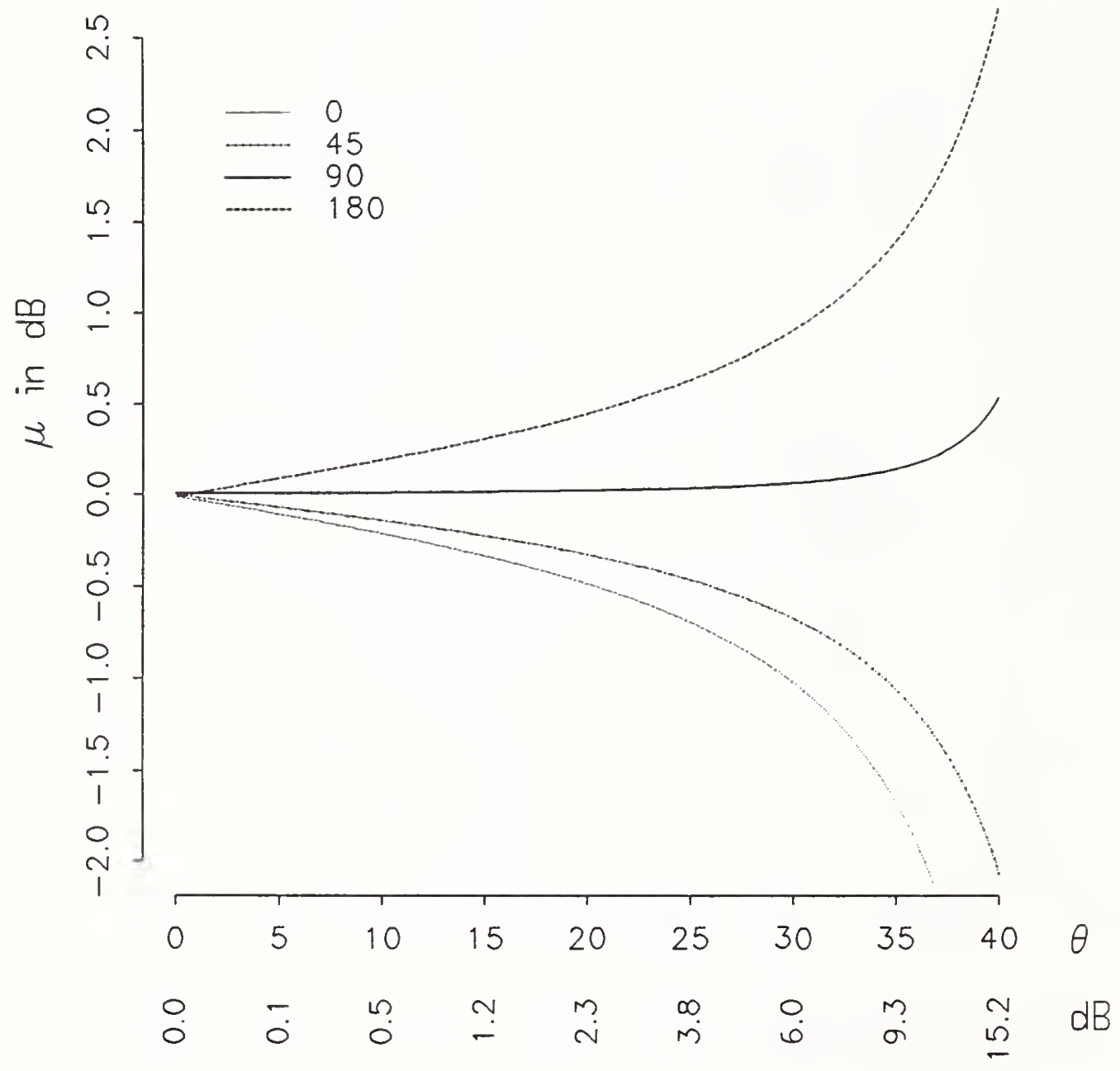

Figure 5: The error $\mu$ in the measured RCS of a dihedral scatterer for $\alpha=0^{\circ}$, $45^{\circ}, 90^{\circ}$, and $180^{\circ}(\eta=30 \mathrm{~dB})$. 


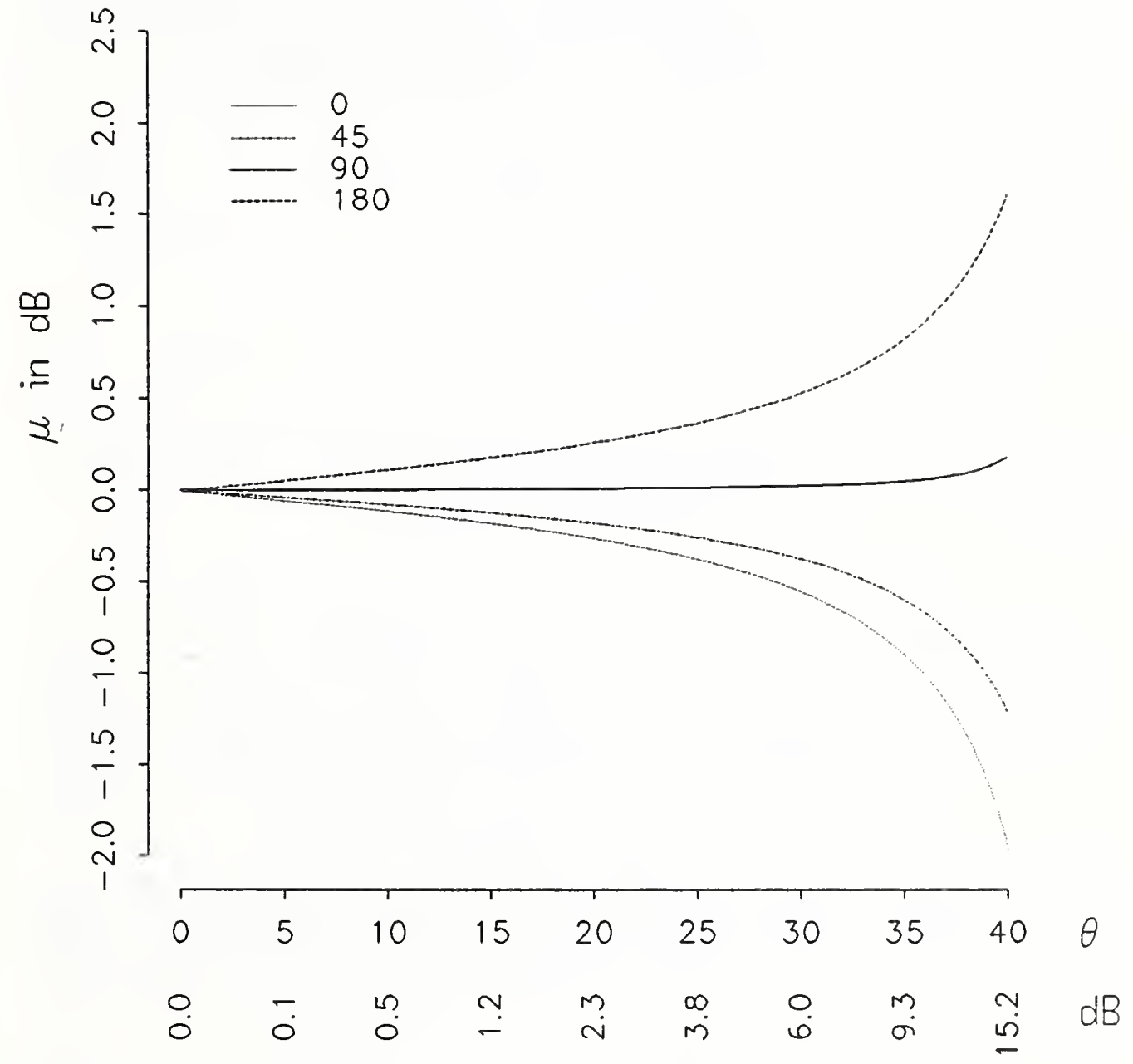

Figure 6: The error $\mu$ in the measured RCS of a dihedral scatterer for $\alpha=0^{\circ}$, $45^{\circ}, 90^{\circ}$, and $180^{\circ}(\eta=35 \mathrm{~dB})$. 
lated [11],

$$
\begin{aligned}
\epsilon_{v} & \equiv R_{v h} / R_{v v}=T_{h v} / T_{v v} \\
\epsilon_{h} & \equiv R_{h v} / R_{h h}=T_{v h} / T_{h h}
\end{aligned}
$$

for the vertical and horizontal polarization ratios, $\epsilon_{v}$ and $\epsilon_{h}$ (see appendix A). Although antenna reciprocity has been considered by other authors $[5$, 12], it has not been fully exploited. With the port-to-port ratios

$$
\begin{aligned}
& \rho \equiv R_{v v} / R_{h h} \\
& \tau \equiv T_{v v} / T_{h h},
\end{aligned}
$$

eq (14) may be written

$$
\left(\begin{array}{cc}
S_{h h} & S_{h v} \\
S_{v h} & S_{v v}
\end{array}\right)=K\left(\begin{array}{cc}
1 & \epsilon_{h} \\
\rho \epsilon_{v} & \rho
\end{array}\right)\left(\begin{array}{cc}
A_{h h} & A_{h v} \\
A_{v h} & A_{v v}
\end{array}\right)\left(\begin{array}{cc}
1 & \tau \epsilon_{v} \\
\epsilon_{h} & \tau
\end{array}\right)
$$

where the complex constant $K$ depends on the target distance. For the dihedral represented by the scattering matrix eq (8) we have, component by component

$$
\begin{aligned}
& S_{h h}(\theta)=K K_{d}\left[2 \epsilon_{h} \sin 2 \theta+\left(1-\epsilon_{h}^{2}\right) \cos 2 \theta\right] \\
& S_{h v}(\theta)=\tau K K_{d}\left[\left(1+\epsilon_{h} \epsilon_{v}\right) \sin 2 \theta-\left(\epsilon_{h}-\epsilon_{v}\right) \cos 2 \theta\right] \\
& S_{v h}(\theta)=\rho K K_{d}\left[\left(1+\epsilon_{h} \epsilon_{v}\right) \sin 2 \theta-\left(\epsilon_{h}-\epsilon_{v}\right) \cos 2 \theta\right] \\
& S_{v v}(\theta)=\tau \rho K K_{d}\left[2 \epsilon_{v} \sin 2 \theta-\left(1+\epsilon_{v}^{2}\right) \cos 2 \theta\right]
\end{aligned}
$$

The first task is to obtain the vertical and horizontal polarization ratios for the radar. (The radar's I/Q output is required to obtain relative phase information.) Treating vertical polarization first, let $y_{v} \equiv S_{v v}\left(0^{\circ}\right) / S_{v v}\left(45^{\circ}\right)$. From eq (21), we obtain

$$
2 y_{v}=\epsilon_{v}-1 / \epsilon_{v} .
$$

The two solutions of eq (22) are negative inverses of each other, and we choose the one with $\left|\epsilon_{v}\right|<1$. For instance, when $\left|\epsilon_{v}\right| \leq \sqrt{2}-1$ we have $\left|y_{v}\right| \geq 1$, so

$$
\epsilon_{v}=y_{v}\left(\sqrt{1+\frac{1}{y_{v}^{2}}}-1\right)
$$


According to eqs (18-21), ideal data should have a simple dependence on rotation angle. In order to reduce the effects of clutter and noise, $S_{v v}(\theta)$ is measured for $0 \leq \theta<360^{\circ}$. We perform a fast Fourier transform (FFT) and retain only coefficients corresponding to an $\exp ( \pm 2 i \theta)$ dependence. This allows construction of a function $\tilde{S}_{v v}(\theta)$ fitting eq (21). The smoothed parameter $\tilde{y}_{v} \equiv \tilde{S}_{v v}(0) / \tilde{S}_{v v}\left(45^{\circ}\right)$ can be used in eq (22) to obtain an improved value for the vertical polarization ratio. Similarly, the horizontal polarization ratio is obtained by constructing the smoothed parameter $\tilde{y}_{h} \equiv-\tilde{S}_{h h}\left(0^{\circ}\right) / \tilde{S}_{h h}\left(45^{\circ}\right)$ and then solving the quadratic equation obtained from eq (22) by substituting $h$ for $v$.

The port-to-port coupling ratios can be determined from smoothed data as, for example,

$$
\rho=\frac{1+2 \epsilon_{h}-\epsilon_{h}^{2}}{1-\epsilon_{h}+\epsilon_{v}+\epsilon_{h} \epsilon_{v}} \frac{\tilde{S}_{v h}\left(22.5^{\circ}\right)}{\tilde{S}_{h h}\left(22.5^{\circ}\right)}
$$

[see eqs $(18,20)]$ and

$$
\tau=\frac{1+2 \epsilon_{h}-\epsilon_{h}^{2}}{1-\epsilon_{h}+\epsilon_{v}+\epsilon_{h} \epsilon_{v}} \frac{\tilde{S}_{h v}\left(22.5^{\circ}\right)}{\tilde{S}_{h h}\left(22.5^{\circ}\right)}
$$

[see eqs $(18,19)]$.

The constant $|K|$ may now be determined from any of eqs (18-21) using a measured or theoretical value [7] for $\left|K_{d}\right|$. The use of smoothed data will reduce clutter and noise contamination. Once the radar is calibrated, a general target's scattering matrix may be found from eq (17), keeping in mind that $K$ must be adjusted to reflect target distance.

Normally, polarimetric calibration can be accomplished by measuring three targets, such as a dihedral oriented at $0^{\circ}$ and $45^{\circ}$, and a trihedral reflector (or sphere) [5,6]. An advantage of our procedure, when it applies ${ }^{3}$, is that a trihedral reflector (or sphere) is no longer needed. The dihedral reflector can be mounted on a rotator (which could possibly be range-gated out) and aligned to rotate about an axis coincident with the radar's boresight axis. For ease of alignment a Bruderhedral reflector [9, 10], which consists of a sector of a cylinder mounted on a flat plate and which has a dihedrallike response but a broader azimuthal pattern, could be substituted for the dihedral reflector.

\footnotetext{
${ }^{3}$ Again, we are considering a reciprocal, dual-port antenna system and perfectly isolated transmitter and receiver channels.
} 


\section{Conclusions}

This paper has examined the error which can occur when a radar is assumed to be ideally polarized. The characterizations 'strong' and 'weak' compare target depolarization to the radar's polarization isolation. For strong depolarization, even with good antenna polarization isolation, errors can be unacceptably large.

Full polarimetric calibration allows correction for cross-polarization errors. A simplified procedure is presented for calibration of reciprocal-antenna radars with isolated receiver and transmitter channels. Here, only a single calibration target (a rotatable dihedral) is required, instead of the two or three required by other methods. The procedure can be used to calibrate radars currently used just for vertical and horizontal polarization measurements. The calibration data can be smoothed by employing Fourier filtering, which should significantly reduce the effects of clutter and noise. 


\section{References}

[1] R. C. Wittmann, M. H. Francis, L. A. Muth, and R. L. Lewis, "Proposed analysis of RCS measurement uncertainty," Antenna Measurement Techniques Association, Proceedings, pp. 51-57, Oct. 3-7, 1994. See also, R. C. Wittmann, M. H. Francis, L. A. Muth, and R. L. Lewis, "Proposed uncertainty analysis for RCS measurements," National Institute of Standards and Technology, NISTIR 5019, Jan. 1994.

[2] R. M. Barnes, "Polarimetric calibrations using in-scene reflectors," MIT Lincoln Lab., Lexington, MA, Rep. TT-65, Sept. 1986.

[3] S. H. Yueh, J. A. Kong, R. M. Barnes, and R. T. Shun, "Calibration of polarimetric radars using in-scene reflectors," J. Electromagn. Wave Appl., vol. 4, pp. 27-48, Jan. 1990.

[4] T. J. Chen, T. H. Chu, and F. C. Chen, "A new calibration algorithm of wide-band polarimetric measurement system," IEEE Trans. Antennas Propagat., vol. AP-39, pp. 1188-1192, August 1991.

[5] M. W. Witt, F. T. Ulaby, P. Polatin, and V. V. Liepa, "A general polarimetric radar calibration technique," IEEE Trans. Antennas Propagat., vol. AP-39, pp. 62-67, Jan. 1991.

[6] M. W. Witt and F. T. Ulaby, "A polarimetric radar calibration technique with insensitivity to target orientation," Radio Science, vol. 25, no. 6, pp. 1137-1143, Nov.-Dec. 1990.

[7] G. T. Ruck, ed., Radar Cross Section Handbook, vol. 2, pp. 588-590. New York: Plenum Press, 1970.

[8] N. C. Currie, ed., Techniques of Radar Reflectivity Measurement, chs. 2 \& 4. Boston: Artech House, 1984.

[9] N. C. Currie and C. E. Brown, eds., Principles and Applications of Millimeter-Wave Radar, ch. 17. Boston: Artech House, 1987.

[10] N. C. Currie, ed., Radar Reflectivity Measurement: Techniques and Applications, chs. 1-4. Boston: Artech House, 1989.

[11] J. A. Scheer and J. L. Kurtz, eds., Coherent Radar Performance Estimation, pp. 266-268. Boston: Artech House, 1993. 
[12] K. Sarabandi and F. T. Ulaby, "A convenient technique for polarimetric calibration of single-antenna radar systems," IEEE Trans. Geosci. Remote Sensing, vol. GE-28, pp. 1022-1033, Nov. 1990. 


\section{A Appendix Reciprocity Relations}

We have assumed that the transmitter/receiver channels are perfectly isolated so that the only cross-polarization contamination is introduced by the reciprocal-antenna system. As shown here, these assumptions lead directly to eqs (15).

The received signal matrix for a fully polarimetric radar can be written in the form

$$
\mathbf{S} \propto \mathbf{R A T}=\boldsymbol{\gamma} \mathbf{R}^{\prime} \mathbf{A} \mathbf{T}^{\prime} \boldsymbol{\beta} .
$$

Here, $\mathbf{R}$ and $\mathbf{T}$ denote the radar system's transmitting and receiving matrices and $\mathbf{A}$ is the target's scattering matrix. $\mathbf{R}^{\prime}$ and $\mathbf{T}^{\prime}$ are the antenna system's transmitting and receiving matrices, and $\boldsymbol{\beta}$ and $\boldsymbol{\gamma}$ are diagonal matrices which represent a transmitter/receiver system with perfect polarization isolation. Now, for a reciprocal, dual-port, radar antenna, we have $\mathbf{R}^{\prime}=\mathbf{T}^{\prime t}$ where the superscript $t$ denotes a matrix transpose. Consequently, it follows that

$$
\mathbf{R}=\boldsymbol{\gamma} \boldsymbol{\beta}^{-1} \mathrm{~T}^{t} .
$$

Here $\boldsymbol{\gamma} \boldsymbol{\delta} \boldsymbol{\beta}^{-1}$ is diagonal, and eqs (15) follow at once. 


\section{B Appendix \\ Polarimetric Calibration of Two-Antenna Radars}

A polarimetric two-antenna radar's received signal matrix is expressed by eq (14) ; however, there is no analytic relationship between the radar system's transmit and receive matrices. Nevertheless, since each antenna is assumed to be reciprocal and since transmit-receive duplexers can be inserted into the antenna feed lines, four independent measurements can be carried out to separately determine each antenna's polarization ratios using eq (22). Carrying out such a polarization calibration for the transmitting antenna produces

$$
\epsilon_{v} \equiv \frac{T_{v h}}{T_{v v}}, \quad \epsilon_{h} \equiv \frac{T_{h v}}{T_{h h}} .
$$

Similarly, for the receiving antenna we obtain

$$
\epsilon_{v}^{\prime} \equiv \frac{R_{v h}^{\prime}}{R_{v v}^{\prime}}, \quad \epsilon_{h}^{\prime} \equiv \frac{R_{h v}^{\prime}}{R_{h h}^{\prime}}
$$

where primes denote the receiving antenna. The transmit-receive duplexers can be removed once the polarization ratios are determined. Using eqs (28) and (29) in eq (14), we obtain

$$
\left(\begin{array}{ll}
S_{h h} & S_{h v} \\
S_{v h} & S_{v v}
\end{array}\right)=K\left(\begin{array}{cc}
1 & \epsilon_{h}^{\prime} \\
\rho^{\prime} \epsilon_{v}^{\prime} & \rho^{\prime}
\end{array}\right)\left(\begin{array}{ll}
A_{h h} & A_{h v} \\
A_{v h} & A_{v v}
\end{array}\right)\left(\begin{array}{cc}
1 & \tau \epsilon_{v} \\
\epsilon_{h} & \tau
\end{array}\right) .
$$

Here the unknown principal component ratios $\rho^{\prime} \equiv R_{v v}^{\prime} / R_{h h}^{\prime}$ and $\tau=T_{v v} / T_{h h}$ need to be determined in order to complete a full polarimetric calibration. Since the left side of eq (30) represents measured data, the unknown ratios are determined if the scattering matrix $\mathbf{A}$ is known. For example, expressing the solution in terms of a dihedral reflector oriented at $22.5^{\circ}$ from horizontal, we obtain

$$
\rho^{\prime}=\frac{1+\epsilon_{h}^{\prime}+\left(1-\epsilon_{h}^{\prime}\right) \epsilon_{h}}{1+\epsilon_{v}^{\prime}-\left(1-\epsilon_{v}^{\prime}\right) \epsilon_{h}} \frac{\tilde{S}_{v h}\left(22.5^{\circ}\right)}{\tilde{S}_{h h}\left(22.5^{\circ}\right)}
$$

and

$$
\tau=\frac{1+\epsilon_{h}+\left(1-\epsilon_{h}\right) \epsilon_{h}^{\prime}}{1+\epsilon_{v}-\left(1-\epsilon_{v}\right) \epsilon_{h}^{\prime}} \frac{\tilde{S}_{h v}\left(22.5^{\circ}\right)}{\tilde{S}_{h h}\left(22.5^{\circ}\right)}
$$


where $\tilde{S}_{x y}(\theta)$ denotes a smoothed signal resulting from retaining only Fourier coefficients from terms with an $\exp ( \pm 2 i \theta)$ dependence. The last remaining unknown $|K|$ is determined as described in section 2 . 

